\title{
Autocorrelation properties of chaotic delay dynamical systems: A study on semiconductor lasers
}

\author{
Xavier Porte, ${ }^{1, *}$ Otti D’Huys, ${ }^{2}$ Thomas Jüngling, ${ }^{1}$ Daniel Brunner, ${ }^{1}$ Miguel C. Soriano, ${ }^{1}$ and Ingo Fischer ${ }^{1}$ \\ ${ }^{1}$ Instituto de Física Interdisciplinar y Sistemas Complejos, IFISC (CSIC-UIB), Campus Universitat Illes Balears, \\ E-07122 Palma de Mallorca, Spain \\ ${ }^{2}$ Institute of Theoretical Physics, University of Würzburg, 97074 Würzburg, Germany
}

(Received 25 June 2014; published 12 November 2014)

\begin{abstract}
We present a detailed experimental characterization of the autocorrelation properties of a delayed feedback semiconductor laser for different dynamical regimes. We show that in many cases the autocorrelation function of laser intensity dynamics can be approximated by the analytically derived autocorrelation function obtained from a linear stochastic model with delay. We extract a set of dynamic parameters from the fit with the analytic solutions and discuss the limits of validity of our approximation. The linear model captures multiple fundamental properties of delay systems, such as the shift and asymmetric broadening of the different delay echoes. Thus, our analysis provides significant additional insight into the relevant physical and dynamical properties of delayed feedback lasers.
\end{abstract}

DOI: 10.1103/PhysRevE.90.052911

PACS number(s): 05.45.Jn, 42.55.Px, 02.30.Ks

\section{INTRODUCTION}

Systems with delayed interactions play a prominent role in a variety of fields, ranging from traffic [1] and population dynamics [2], gene regulatory [3] and neural networks [4], and encrypted communications [5]. When subjecting a semiconductor laser to reflections of its own emission, a delay results from the propagation time of the light in the external cavity. Because of its experimental accessibility and multiple applications, semiconductor lasers with delayed feedback or coupling have become one of the most studied delay systems [6].

In semiconductor lasers, delayed feedback can induce high-dimensional chaos $[7,8]$. In experiments, one of the most accessible properties to characterize intensity dynamics is the autocorrelation function (AC). Particular characteristics of chaotic attractors, such as Lyapunov exponents, entropy, or correlation dimensions, are often hard or impossible to obtain experimentally due to the high dimensionality and noise in the system. The relationship between the AC and other nonlinear properties of the system is, however, generally unknown and can be regarded as nontrivial except for a few cases $[9,10]$. Therefore, although the AC is often one of the key characteristics measured, it is unclear which information can be extracted from it.

The AC of time series from a delayed feedback laser clearly shows the fingerprint of the delay time, with extrema around multiples of the time delay. However, these delay echoes do not always appear exactly at the delay time but show an additional shift, often associated with an internal time scale [11-15]. In this paper we introduce a linear stochastic model with delay, which allows for analytic calculation of the autocorrelation function. We systematically compare the $\mathrm{AC}$ of our linear model with the experimental $\mathrm{AC}$ of a semiconductor laser subject to delayed optical feedback. We investigate which characteristic features of the experimentally observed AC can be explained by this linear model and, therefore, do not originate from the nonlinear response of the system. Despite

*Corresponding author: xavier@ifisc.uib-csic.es its simplicity, we find that in some dynamical regimes the experimentally observed AC can be approximated by this model surprisingly well. We show that our model offers a general perspective on chaotic delay dynamics of lasers on a high level of detail throughout a broad range of dynamical regimes.

The paper is organized as follows. We first present typical characteristics of the autocorrelation function of delay systems, using the semiconductor laser with delayed feedback as an illustration. In Sec. III, we motivate and introduce our linear stochastic model with delay and analytically calculate its autocorrelation function. We describe our experiments in Sec. IV and compare the experimental semiconductor laser AC to its linear counterpart. By means of numerical simulations and analytical approximations we relate the derived parameters of the AC to physical properties of the laser. Finally, we discuss the validity of our approximation in detail.

\section{AUTOCORRELATION FUNCTION OF DELAY SYSTEMS}

For delay systems the autocorrelation function typically shows not only a decaying central peak but also signatures around multiples of the delay time which we denote as delay echoes. In the case of the $\mathrm{AC}$ originating from intensity time series of a laser experiment, the delay echoes exhibit a certain peak structure, indicating the response of the laser to the perturbation of the delayed feedback signal [16]. An exemplary $\mathrm{AC}$ of a semiconductor laser with optical feedback is presented in Fig. 1(a), with a central peak around zero and a number of identifiable delay echoes. The decay rate of these structures, and hence the number of visible delay echoes, depends on the experimental conditions [17]. Figure 1(b) shows a magnification of the central peak structure and the two first delay echoes shifted by their respective multiples of the feedback delay. The external cavity round-trip time $\tau$ was accurately measured independently of the semiconductor laser dynamics (as explained in Sec. IV), and thus, we know the precise position of these peaks relative to the delay. The dashed curves in Fig. 1(b) represent the envelopes of the consecutive AC delay echoes, which are calculated using the Hilbert transform [18]. As it can be seen, successive echoes 

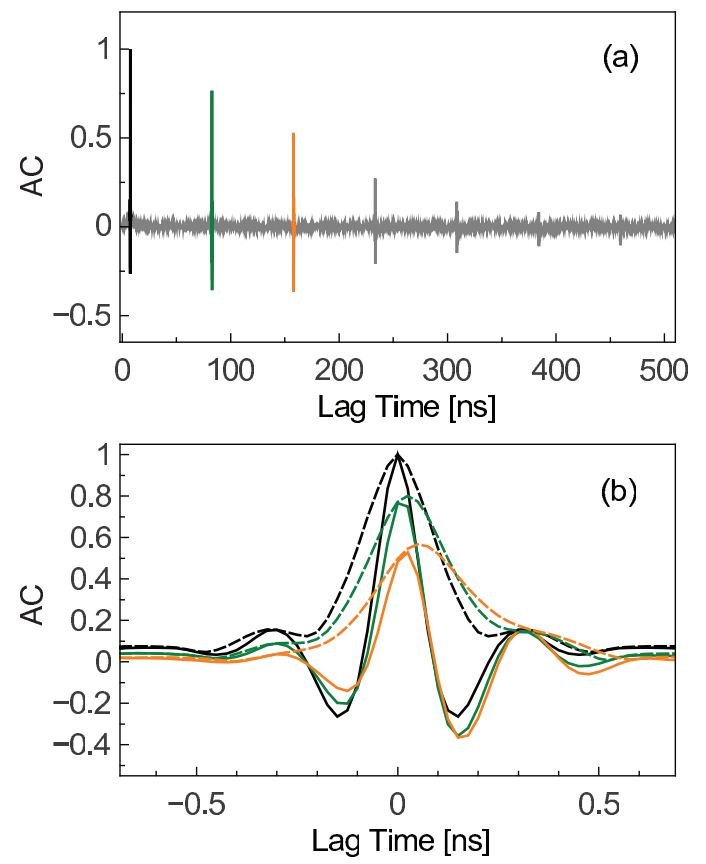

FIG. 1. (Color online) (a) AC function showing multiple delay echoes. The current and attenuation are $13 \mathrm{~mA}$ and $6.1 \mathrm{~dB}$, respectively. Panel (b) is a zoom into the highlighted central (black), first (dark color), and second (light color) delay echoes. The AC (full lines) and envelope (dashed lines) echoes have been shifted by a temporal offset equal to their respective multiples of the delay time.

accumulate a phase shift with respect to the symmetric central peak. In addition to the phase shift in the successive AC delay echoes, there is an offset in the position of the envelope maxima with respect to the exact delay time. Moreover, there is a noticeable asymmetric broadening of the envelopes of the delay echo.

\section{AUTOCORRELATION FUNCTION OF A LINEAR STOCHASTIC DELAY MODEL}

From a theoretical point of view, a laser subject to feedback is a nonlinear system driven by its own time-delayed variables. The response of the laser is a nonlinear transformation of the incoming signal, giving rise to the observed chaotic dynamics. The role of the nonlinearity in the creation of the typical trajectories on the chaotic attractor depends on the dynamical regime and hence on parameters. One might, for instance, expect different processes for strong or weak chaos [19]. However, in case of the laser diode, typical transient oscillations have a duration of several delay times, while the distortions between consecutive delay iterations can be considered as minor. Therefore, the active nonlinearity can be regarded as weak. The delay system as a whole generates very complex waveforms which have been employed for, e.g., random number generation [20-23].

In the following, we approximate the complex driving signal as noise, which is a common procedure for fast chaotic dynamics [24]. Although we have a clear time scale separation between the delay time and the characteristic time scales of the laser, e.g., expressed by relaxation oscillations frequency and damping, the modeling approach presented here differs from the one in Ref. [24]. Our main idea consists in the extension of a linear response theory to the fully developed chaotic regime. In particular, we demonstrate that the experimentally and numerically obtained AC of a chaotic laser can be largely reproduced by a simple linear oscillator with delayed feedback excited by noise [25].

Such a model reflects emergent properties of the underlying complex dynamics rather than representing the equations of motion in the usual sense. The direct comparison of model and the original dynamics concentrates on the level of the AC. Properties like dimensionality and the Lyapunov spectrum are not preserved, similarly to surrogate analysis. Nevertheless, the quality and extent of the modeling suggests that this approach captures dominant features of the time series, thus allowing for an appropriate description of complex dynamics as well as qualitative and even quantitative insight into physical properties of the system.

Without delay, a linear model results in an AC decaying exponentially as $\cos (\omega t) e^{-\alpha t}$. Such an exponential decay is not uncommon for (undelayed) chaotic systems, specifically for so-called spiral attractors [10]. More generally, the AC for a wide class of chaotic attractors can be written as a sum of decaying exponentials. The relation between these decay rates (and their corresponding frequencies) and the dynamical parameters of the chaotic system is in many cases still an open problem. Moreover, the AC of different system variables do not necessarily decay in the same way.

We focus on the influence of the delay: We calculate the shape of the delay echoes explicitly and show how the dynamics of a delay interval is, on average, transformed during each round-trip by linear delay effects only. We consider a simple linear stochastic oscillator with delayed feedback, modeled via

$$
\dot{x}(t)=(-\alpha+i \omega) x(t)+\beta e^{-i \phi} x(t-\tau)+\xi(t),
$$

with $x \in \mathbb{C}$. The parameter $\alpha>0$ represents the characteristic damping of the oscillator and $\omega$ is its characteristic frequency. The delayed feedback is characterized by a strength $\beta<\alpha$, a feedback phase $-\phi$, and a long feedback delay $\tau \gg$ $\max \left(\alpha^{-1}, \beta^{-1}, \omega^{-1}\right)$. The time scale separation between delay time and the oscillator parameters is essential in order to adapt to the experimental conditions and reflect the properties behind the clearly separated autocorrelation signatures as shown in the previous section. The oscillator is driven by white noise $\xi(t)$ with $\left\langle\xi(t) \xi\left(t^{\prime}\right)\right\rangle=2 D \delta\left(t-t^{\prime}\right)$. In comparison with the experimental system, $x(t)$ in the linear oscillator is associated with the time-dependent intensity of the laser, whereas $\omega$ and $\alpha$ are interpreted as the relaxation oscillations frequency and damping, respectively. The driving noise being white is an idealization which takes into account the high bandwidth of the typical chaotic trajectories. It can be further understood to incorporate hidden degrees of freedom, in particular the dynamics of the optical phase, which we have not measured simultaneously with the intensity, as well as the intrinsic noise of the experimental system.

To compute the (complex) autocorrelation function

$$
r(k)=\frac{\left\langle x(t+k) x^{*}(t)\right\rangle}{\left\langle|x|^{2}(t)\right\rangle},
$$


we evaluate Eq. (1) at $t=t_{0}+k$. By multiplying with $x^{*}\left(t_{0}\right)$ and averaging, we obtain, after normalization, a closed equation for the autocorrelation function $r(k)$,

$$
\dot{r}(k)=(-\alpha+i \omega) r(k)+\beta e^{-i \phi} r(k-\tau),
$$

where the derivative is now with respect to $k$. Equation (2) can be integrated in the different delay intervals. For the first interval $\left[0, \tau\right.$ [ we choose as initial conditions $r_{0}(k)=r_{0}^{*}(-k)$ and $\lim _{\tau \rightarrow \infty} r_{0}(0)=1$. This leads to a solution

$$
r_{0}(k)=e^{(-\Lambda+i \omega) k}+\gamma e^{(\Lambda+i \omega)(k-\tau)} .
$$

The envelope of the autocorrelation function $\left|r_{0}(k)\right|$ thus decays exponentially for $k \approx 0$ and increases with the same exponent $\Lambda$ as $k \rightarrow \tau$ from the left-hand side. The oscillation frequency of both the central peak and the first delay peak is given by the characteristic frequency $\omega$. The previously mentioned decay/growth rate $(\Lambda)$ is given by

$$
\Lambda=\sqrt{\alpha^{2}-\beta^{2}},
$$

i.e., it depends on the real part $\alpha$ of the characteristic dynamics and on the magnitude $\beta$ of the feedback. The amplitude of the first delay peak $\gamma$ reads

$$
\gamma=\frac{\beta e^{-i \phi}}{\Lambda+\alpha}
$$

leading to a phase shift of the first peak at $k=\tau$ equal to the feedback phase $-\phi$.

In the interval $[\tau, 2 \tau$ [ the autocorrelation follows the equation

$$
\dot{r}_{1}(k)=(-\alpha+i \omega) r_{1}(k)+\beta e^{-i \phi} r_{0}(k-\tau) .
$$

To preserve continuity we impose as initial condition $\lim _{\tau \rightarrow \infty} r_{1}(\tau)=\gamma$. We find a solution

$$
\begin{aligned}
r_{1}(k)= & \gamma^{*-1} e^{(-\Lambda+i \omega)(k-\tau)}+\gamma^{2} e^{(\Lambda+i \omega)(k-2 \tau)} \\
& +\left(\gamma-\gamma^{*-1}\right) e^{(-\alpha+i \omega)(k-\tau)} .
\end{aligned}
$$

Thus, the first delay echo is asymmetric with $r_{0}(\tau-k) \neq$ $r_{1}(\tau+k)$ : The right side is broadened. Moreover, neither the highest peak, nor the envelope of the first delay echo are maximal at $k=\tau$. The oscillation frequency within the first delay echo also corresponds to the characteristic frequency of the system.

In a similar way the autocorrelation function can be integrated in the consecutive delay intervals. In the interval $\left[n \tau,(n+1) \tau\right.$ [ we find an autocorrelation $r_{n}(k)$, given by

$$
\begin{aligned}
r_{n}(k)= & \gamma^{*-n} e^{(-\Lambda+i \omega)(k-n \tau)}+\gamma^{n+1} e^{(\Lambda+i \omega)(k-(n+1) \tau)} \\
& +\left[\gamma^{n} \sum_{m=0}^{n} \frac{\left(\gamma^{-1} \beta e^{-i \phi}(k-n \tau)\right)^{m}}{m !}\right. \\
& \left.-\gamma^{*-n} \sum_{m=0}^{n} \frac{\left(\gamma^{*} \beta e^{-i \phi}(k-n \tau)\right)^{m}}{m !}\right] e^{(-\alpha+i \omega)(k-n \tau)} .
\end{aligned}
$$

Each delay peak is more asymmetrically broadened than the previous one, and the maxima are shifted further away from $k=n \tau$. The envelope, the real part of the central peak, and the first two delay echoes are shown in Fig. 2.

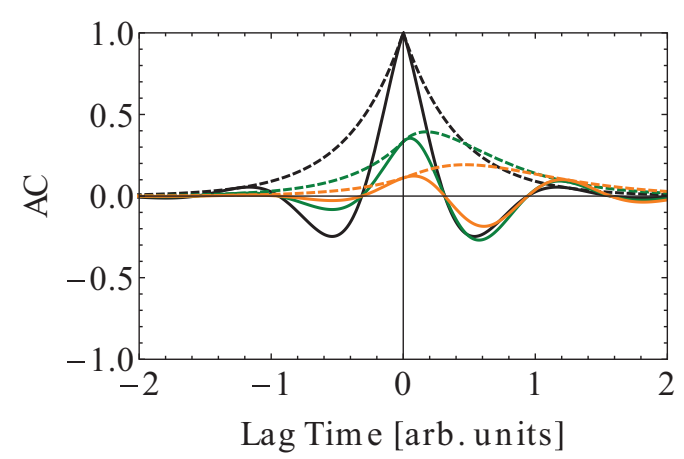

FIG. 2. (Color online) Envelope (dashed lines) and real part (full lines) of the central peak and the first two delay peaks of the autocorrelation function of a linear stochastic model [Eq. (1)]. Parameters are $\alpha=3, \beta=1.8, \omega=5, \phi=0$, and $\tau \rightarrow \infty$. The broadening of the consecutive peaks and the shift of the maximum are clearly visible.

Interestingly, multiple characteristic features observed in the $\mathrm{AC}$ of the chaotic feedback laser are captured by this simple linear model. Our model reproduces the shift and the broadening of the delay echoes. These features therefore are not necessarily induced by the instantaneous nonlinear transformation of the delayed signal by a chaotic element, but might represent generic features of the AC of delay dynamical systems. In particular, the shift of the first envelope can be analytically derived as follows:

$$
k_{\text {shift }}=\frac{1}{\alpha-\Lambda} \ln \frac{2 \alpha}{\Lambda+\alpha}=\frac{1}{2 \alpha}\left[1+\frac{1}{8} \frac{\beta^{2}}{\alpha^{2}}+\mathcal{O}\left(\frac{\beta^{4}}{\alpha^{4}}\right)\right] .
$$

The envelope shift could be linked to the so-called internal time scale [11-15]. We find here that this shift depends not only on the internal parameter $\alpha$ but also on the coupling strength $\beta$. However, for sufficiently weak coupling the envelope shift is indeed proportional to the inverse internal damping rate. The delay echoes are thus shifted due to the combination of two different effects. The oscillation phase of the echoes is shifted due to the presence of a coupling phase $-\phi$. The shift and broadening of the envelope is a universal effect in delay systems and depends on the internal time scale $\alpha^{-1}$ and the ratio between internal an coupling parameter $\frac{\beta}{\alpha}$.

The linear model can easily be extended to multiple characteristic frequencies or colored noise, which would lead to a more accurate approximation of the AC of a chaotic feedback laser. However, here we focus on the simplest case. Since this model contains only a few parameters, direct comparison with chaotic feedback lasers and determination of the parameters remain straightforward.

\section{SEMICONDUCTOR LASER EXPERIMENTS}

We compare the predicted AC features of the linear model with experimental autocorrelation functions of the emission dynamics of a semiconductor laser with delayed optical feedback. For our experiments, we have used a quantum-well discrete-mode (DM) semiconductor laser emitting at $1543 \mathrm{~nm}$. The solitary DM laser has a side-mode suppression ratio of more than $40 \mathrm{~dB}$. The laser current and temperature 


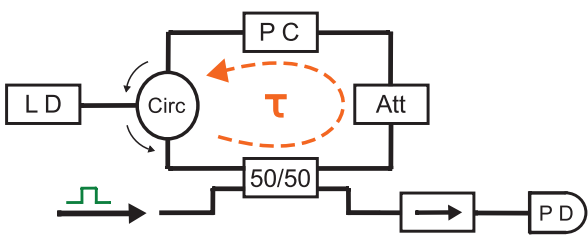

FIG. 3. (Color online) Scheme of the experimental setup. LD, laser diode; Circ, optical circulator; PC, polarization controller; Att, optical attenuator; 50/50, two-by-two 3-dB coupler; $\rightarrow$, optical isolator; and PD, photodiode. The secondary incoming port of the two-by-two coupler is used to inject a train of short pulses to precisely measure the delay time (i.e., external cavity round-trip time, here indicated by a dashed arrow).

are stabilized to an accuracy of $0.01 \mathrm{~mA}$ and $0.01 \mathrm{~K}$, respectively. We subject the laser to polarization maintained optical feedback from a fiber-optic external cavity. Figure 3 depicts a scheme of the experimental fiber based setup. The fiber pigtail of the laser diode is directly attached to an optical circulator to create a fiber loop mirror. We use a two-by-two 3-dB coupler to couple out half of the light for detection.

The intensity time series are acquired using a photodiode with $12.5-\mathrm{GHz}$ bandwidth and a $16-\mathrm{GHz}$ analog bandwidth oscilloscope with a sampling rate of $40 \mathrm{GS} / \mathrm{s}$. The other components within the fiber loop are used to control the polarization (PC) and the intensity (Att) before the light is finally reinjected into the laser diode.

We estimate a maximum feedback rate of $\kappa_{\max }=56 \pm$ $6 \mathrm{~ns}^{-1}$ in our setup. This rate corresponds to the feedback coefficient as defined in Eq. (10). This estimation results from considering all the measured losses of the components and the laser-to-fiber coupling efficiency. From this maximum value, the strength of the feedback can be attenuated by more than $20 \mathrm{~dB}$. The attenuation is then defined as

$$
\text { Attenuation }[\mathrm{dB}]=-20 \log _{10}\left(\frac{\kappa}{\kappa_{\max }}\right) \text {. }
$$

The experimental setup has been designed to allow for a dynamics independent measurement of the external cavity round-trip time by using the additional input port of the two by two coupler. We generate a train of light pulses (represented by the injected square pulse in Fig. 3) which have a duration of $1 \mathrm{~ns}$ each pulse and an interpulse time much larger than the external cavity round-trip time. The pulses are then injected into the external cavity while the laser is turned off and the delay between successive reflections from the laser facet, i.e., the feedback delay time, is measured. Using this method, we find a delay time of $\tau=75.25 \pm 0.025 \mathrm{~ns}$.

As shown in Fig. 1, the AC of the semiconductor laser dynamics shows a characteristic peak structure. The shape and height of the AC around each delay echo strongly depends on the dynamical regime and hence on the experimental conditions. In previous works [17,19] we showed that for large delays this dependency can be characterized to a large extent by a single parameter, namely the feedback (coupling) strength, rescaled by the square root of the current above lasing threshold. Figure 4 depicts the dependence of the maximum of the AC around the first delay echo for four different pump currents as a function of the rescaled attenuation of

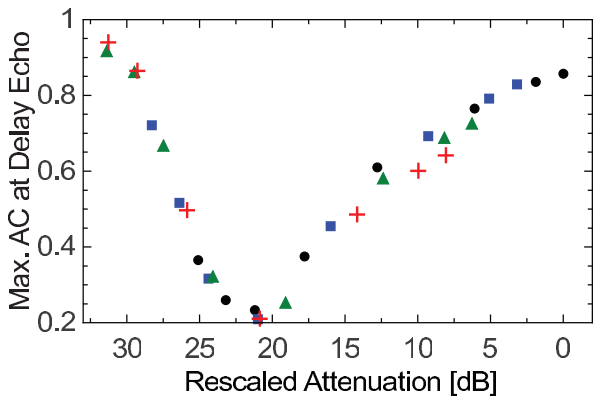

FIG. 4. (Color online) Maximum of the first delay echo of the AC versus the rescaled attenuation for four different operating currents: circles $(I=13 \mathrm{~mA})$, squares $(I=14 \mathrm{~mA})$, triangles $(I=16 \mathrm{~mA})$, and crosses $(I=18 \mathrm{~mA})$.

the feedback signal,

$$
\begin{aligned}
\text { Rescaled Atten. }[\mathrm{dB}]= & -20 \log _{10}\left(\frac{\kappa}{\kappa_{\max }}\right) \\
& +10 \log _{10}\left(\frac{I}{I_{t h}}-1\right) .
\end{aligned}
$$

After rescaling, all four measurements collapse to the same characteristic nonmonotonous curve, with high correlations for low and high feedback strengths and low correlations for moderate feedback strengths.

In order to compare the linear model and the observed AC of the dynamics of a semiconductor laser subject to delayed feedback, we fit the analytically obtained expressions to the experimental results. In particular, Eq. (3) has been fitted to the experimental data using a least-squares method. From the central peak, we fit the parameters $\Lambda$ and $\omega$. From the left side of the first delay echo, we fit the parameters $\beta$ and $\phi$, which are related to $\gamma$ as shown in Eq. (5). This equation, which only describes the AC in the first delay interval, is sufficient to extract all parameters contained in the model and to reproduce the full AC. For parameter extraction, we fit the $\mathrm{AC}$ in two different intervals around the delay echoes, $\left[0, \frac{\tau}{10}\right]$ and $\left[\tau-\frac{\tau}{10}, \tau\right]$, which cover the AC oscillations for most of the experimental conditions.

Figure 5 illustrates the closed approximation of the experimental AC (solid lines) by the analytic curves (dashed lines). Figure 5(a) depicts the central peak structure, and Figures 5(b) and 5(c) the first and second delay echoes of the AC, respectively. The $x$ axis of each panel has been shifted by a time corresponding to its multiple of the feedback delay. The fitted curves correspond to Eq. (3) for the right half of Fig. 5(a) and the left half of Fig. 5(b), and the other analytic curves in Figs. 5(b) and 5(c) correspond to Eqs. (7) and (8), plotted with the parameters obtained from the fit with Eq. (3). The small, yet visible, differences in amplitudes between the analytic curves and the experimental AC is studied in detail in Sec. V.

The same fitting and parameter extraction procedure is applied to the various feedback conditions that we can address in our experiment. We plot in Fig. 6 the extracted model parameters [26] versus the rescaled feedback attenuation [see Eq. (9)]. The curves collapse onto each other, indicating that 


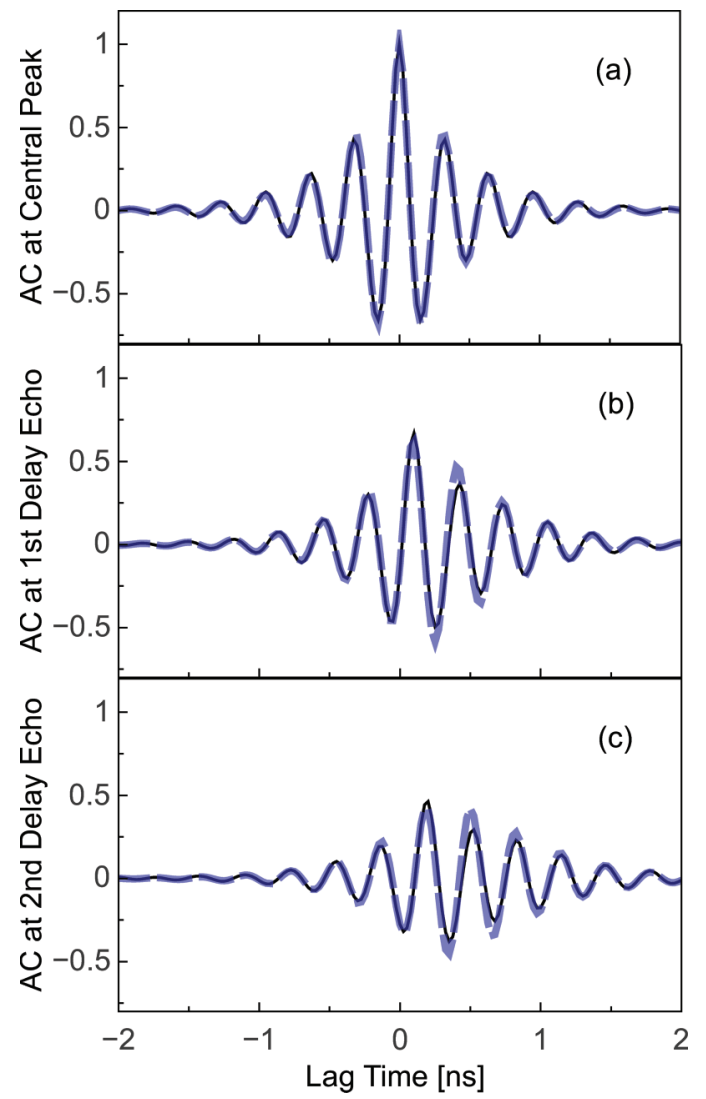

FIG. 5. (Color online) The central peak (a) and the first two delay echoes [(b) and (c), respectively] of the AC of a semiconductor laser subject to delayed feedback for a pump current of $I=16 \mathrm{~mA}$ and a rescaled attenuation of $27.5 \mathrm{~dB}$ with respect to the maximum feedback. The solid lines represent the experimental data and the dashed lines correspond to the fitted analytic expressions for the linear stochastic model.

the linear model correctly fits the self-similar AC curves. Only the extracted frequencies $\frac{\omega}{2 \pi}$ (a) diverge for high feedback strengths; the underlying reason will be explained in Sec. V.

It is known that an increase in feedback strength results in an enlargement of the dynamical bandwidth for most conditions [27,28]. As a consequence, the decay of the central peak of the AC is faster, which is reflected in higher damping parameters $(\Lambda$ and $\alpha)$. The feedback strength of the linear model $(\beta)$ is extracted from the height of the AC at the first delay echo via the second term in Eq. (3). However, it does not grow proportionally to the $\mathrm{AC}$ maximum, which depends on the ratio $\beta / \alpha$ [see Eq. (5)]. The extracted frequency $\frac{\omega}{2 \pi}$ [Fig. 6(a)] approximately corresponds to the dominant frequency component in the radio-frequency spectral density of the laser. In fact, it approaches the relaxation oscillations frequency as the feedback vanishes.

The internal decay parameter $\alpha$ can be related to the damping rate of the relaxation oscillations [29]. In order to support this interpretation, we have repeated the entire set of data analysis presented here for trajectories obtained by simulations of the Lang-Kobayashi (LK) model [30]. The

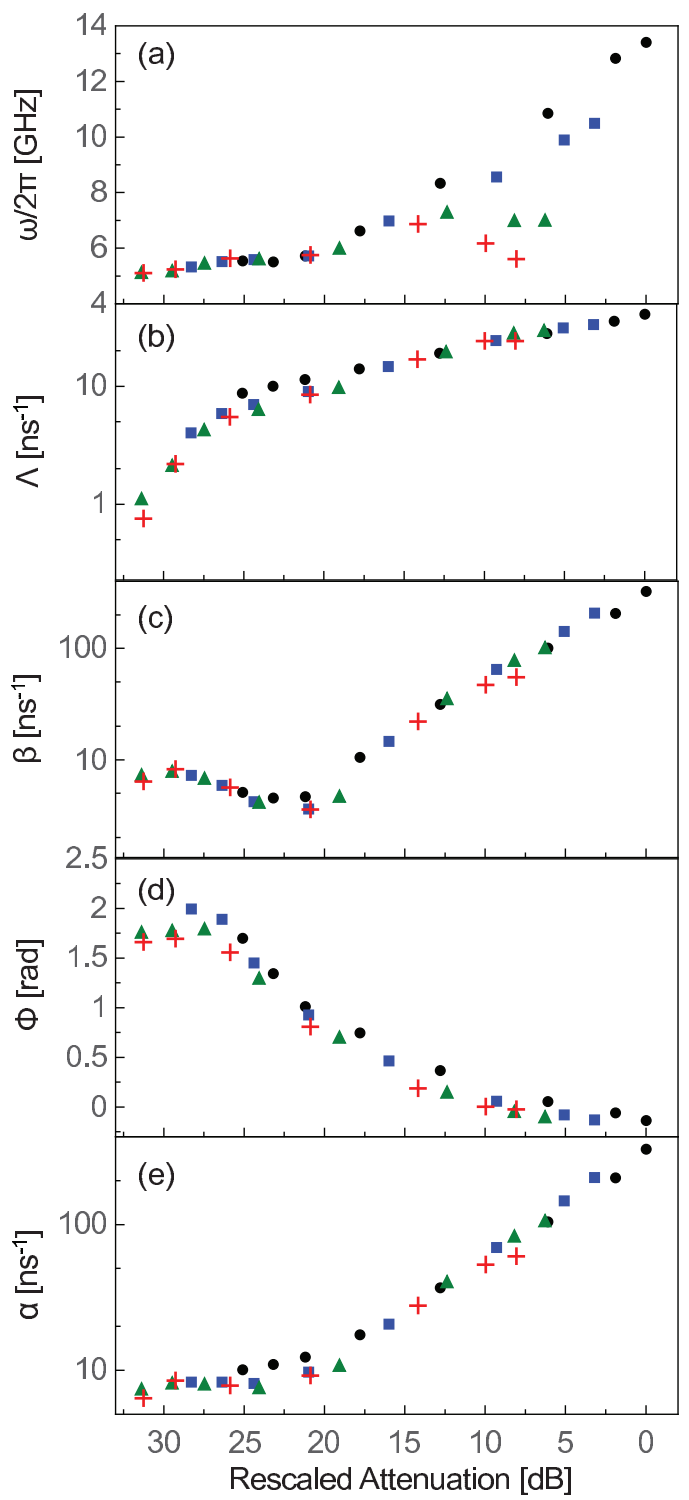

FIG. 6. (Color online) Plot of the rescaled fitted parameters for the different experimental conditions. Panel (a) depicts the internal frequency $\left[\frac{\omega}{2 \pi}\right]$, (b) the decay that includes the effect of the feedback term $[\Lambda]$, (c) the feedback strength $[\beta]$, (d) the feedback phase $[\phi]$, and (e) the internal decay $[\alpha]$. The injection currents associated with each curve are as follows: circles $(I=13 \mathrm{~mA})$, squares $(I=14 \mathrm{~mA})$, triangles $(I=16 \mathrm{~mA})$, and crosses $(I=18 \mathrm{~mA})$.

delayed feedback semiconductor laser can be modeled via

$$
\begin{aligned}
& \dot{\mathcal{E}}(t)=\frac{1+\mathrm{i} \alpha_{H}}{2} G_{N} n(t) \mathcal{E}(t)+\kappa \exp \left(-i \omega_{0} \tau\right) \mathcal{E}(t-\tau) \\
& \dot{n}(t)=(p-1) J_{\mathrm{th}}-\gamma n(t)-\left[\Gamma+G_{N} n(t)\right]|\mathcal{E}(t)|^{2},
\end{aligned}
$$

where $\mathcal{E}(t)$ and $n(t)$ denote the complex electric field and the excess carrier density, respectively. The relative pump current $p$, delay time $\tau$, and feedback strength $\kappa$ have been adapted to the conditions in our experiment. A table of the used parameters can be found in Appendix A. The AC functions of the numerical simulations and the extracted linear model parameters show the same behavior to a large 


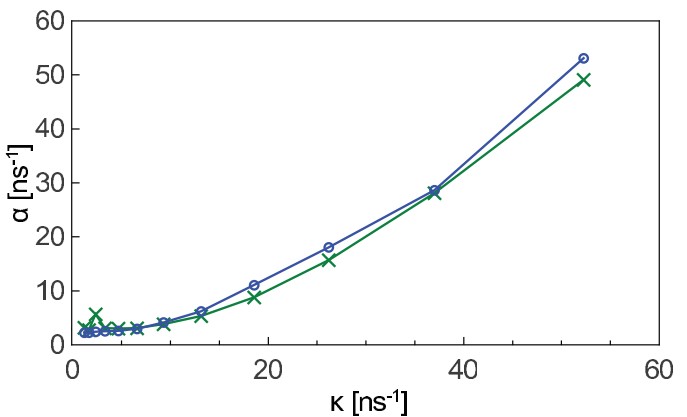

FIG. 7. (Color online) Comparison of $\alpha$ from the AC (green crosses) and $\tilde{\alpha}$ from the linear response approximation (blue circles). The parameters for the simulations are listed in Table I.

extent, as obtained by the experimental trajectories (see also Appendix A).

In contrast to the experiments, numerical simulations allow for access to all system variables, and the equations of motion are explicitly given for all operating conditions. Thus, an independent alternative calculation of a similar damping coefficient $\tilde{\alpha}$ is, in principle, possible: We approximate the LK equations, by replacing the delayed feedback with a noisy drive. In this way, phase and intensity of the laser are decoupled such that the model can be simplified to a driven rate equation model,

$$
\begin{aligned}
& \dot{I}(t)=G_{N} n(t) I(t)+F(t) \\
& \dot{n}(t)=(p-1) J_{\mathrm{th}}-\gamma n(t)-\left[\Gamma+G_{\mathrm{N}} n(t)\right] I(t),
\end{aligned}
$$

with $I(t)=|\mathcal{E}(t)|^{2}$ being the intensity of the laser. In our approximation, the drive $F(t)$ [see Eqs. (B1)] first changes the effective operating point of the laser which is defined by the average intensity and excess carrier density $\left(I_{0}, n_{0}\right)$ and, second, causes local perturbations (relaxation oscillations) around this operating point. The decay rate $\tilde{\alpha}$ of these perturbations is then derived via a linearization of Eq. (11),

$$
\tilde{\alpha}=\left[\gamma-G_{N}\left(n_{0}-I_{0}\right)\right] / 2 .
$$

Based on numerical simulations of Eq. (10), we can then extract decay rates $\alpha$ and $\tilde{\alpha}$ using the linear model and Eq. (12), respectively. Figure 7 depicts both versus the feedback rate, showing very good agreement, supporting the interpretation of $\alpha$ as an extended local damping rate. For a solitary laser, we recover the damping rate of the relaxation oscillations. Both $\tilde{\alpha}$ and $\alpha$ coincide in their definition as the integral over the complex dynamics, which ignores the part of the external cavity and reveals an average response feature of the laser diode.

The AC phase $\phi$ can be interpreted considering the properties of the external cavity modes (ECMs) for the case of weak feedback. External cavity modes are the limit cycle solutions of Eqs. (10) with constant intensity and can be considered as the starting points for the development of different chaotic regimes of the semiconductor laser. The modes destabilize as the feedback increases [31], giving rise to undamped relaxation oscillations around the ECM. For weak feedback it can be assumed that the dynamics is dominated by these oscillations. We associate the AC phase $\phi$ with the phase shift $\varphi$ between the intensity oscillations and its delayed counterpart at the bifurcation point. We assume that the dynamics oscillates

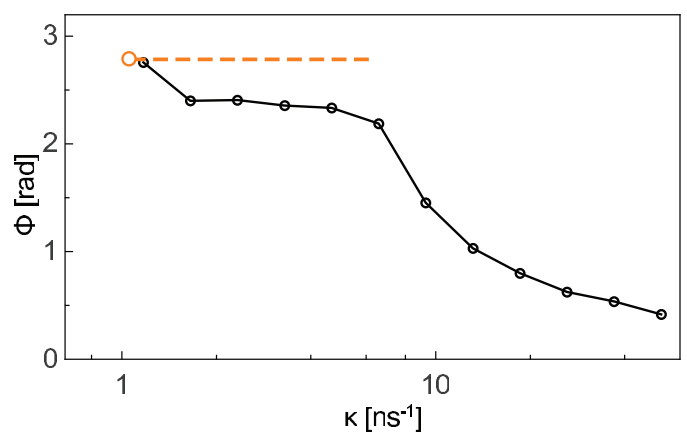

FIG. 8. (Color online) Phase shift $\phi$ extracted from the AC fit (black circles). The dashed line corresponds to the prediction of the phase ( $\varphi$, Appendix B), using a pseudocontinuous spectrum approach at the undamping point.

around the external cavity mode with minimum linewidth, which is known to be the dominant mode when the feedback is weak [29]. The details of this calculation can be found in Appendix B. Figure 8 shows the AC phase $\phi$ of the simulated LK model for the parameters listed in Table I. At low feedback strengths the phase $\phi$ converges to the value of the undamping phase shift $\varphi$ (see Appendix B) of the minimal linewidth mode, represented with the circle at the beginning of the dashed line.

We observe that the shift of the maxima of the delay echoes originates to a large extent from the AC phase shift $\phi$. This is particularly interesting since the delay time is a key parameter to be determined in experimental delay systems [32-41]. We confirm that for low and intermediate feedback strengths the maximum of the delay echo is not a precise indicator of the delay time. We compare in Fig. 9 the shift of the maximum of the first delay echo with the shift of the maximum solely caused by the phase shift $\phi / \omega$. We find a good agreement between the measured shift and the phase-induced shift according to the linear model. Hence, the shift of the envelope contributes only little to the shift of the AC peak at the delay echo. As a consequence, it might be possible to estimate the real delay time with help of the linear model.

\section{VALIDITY OF THE LINEAR MODEL}

In the previous sections we have illustrated the accuracy of a stochastic linear model in reproducing the experimental AC

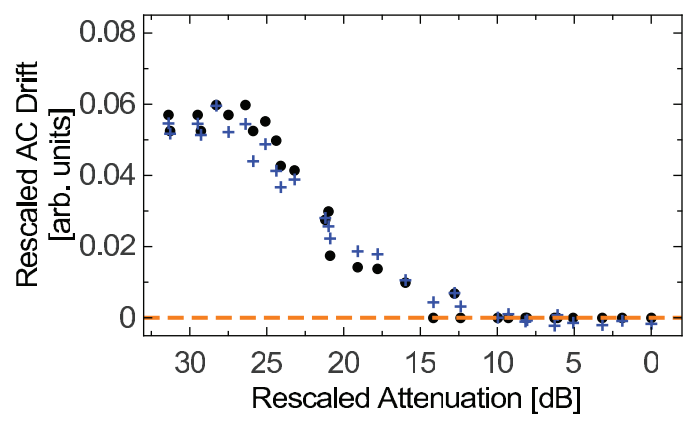

FIG. 9. (Color online) Rescaled shift of the maximum of the AC at the first delay echo for the full set of our experimental conditions. Dots correspond to the shift directly measured from the AC of the experimental time series, and crosses correspond to the shift calculated via the extracted parameters. 


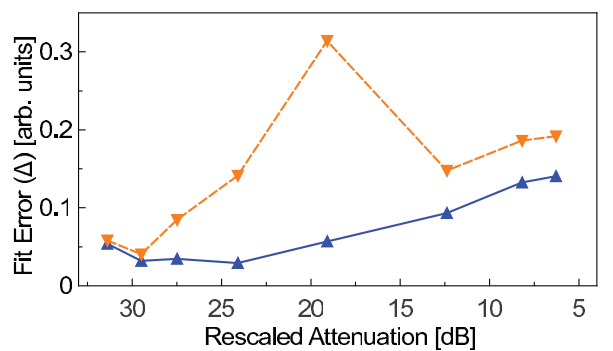

FIG. 10. (Color online) Normalized fitting error of the experimental AC function for a current of $16 \mathrm{~mA}$. The solid line and the dashed line represent the errors for the $\mathrm{AC}$ center and for the first delay echo, respectively.

of the dynamics of a laser subject to optical feedback. Furthermore, the correspondence between model and experiments is supported by the collapsing curves of the fitted parameters for different injection currents, confirming the scaling behavior of the experimental AC [17].

However, the diversity of dynamical regimes present in the experiments has a major influence on the shape of the full AC. Therefore, we quantify the quality of the AC fit using the least-squares error as follows:

$$
\Delta=\frac{1}{\max \left(y_{i}\right)} \sqrt{\frac{\sum_{i=1}^{n}\left[y_{i}-f_{i}(\bar{a})\right]^{2}}{n}},
$$

where $f$ and $y$ represent the analytic results and the experimental data, respectively, and $\bar{a}$ are the parameters obtained from the fit. Errors have been calculated independently for the different delay echoes in windows centered around each peak structure. The widths $n$ of the integration windows were chosen as 10 times the inverse of the decay rate $\Lambda$ of the central envelope. Finally, given the diversity in AC maxima, the errors have been normalized by the maximum height of the $\mathrm{AC}$ at their correspondent peak structure.

Figure 10 depicts the normalized fitting error $\Delta$ for an exemplary current of $16 \mathrm{~mA}$. We highlight two main observations: First, the accuracy of the fit degrades with increasing feedback strength and, second, the errors are smaller for the AC central peak than for the first delay echo, which can be partly related to our fitting procedure.

The region of intermediate feedback strengths exhibits a pronounced increase of the error of the first delay echo (with the maximum at $19.1 \mathrm{~dB}$ in Fig. 10), coinciding with the region of strong chaos in delayed feedback semiconductor lasers [19,42]. Figure 11 illustrates the origin of the large error for this range of feedback strengths. Figures 11(a) to 11(c) show the central peak structure and first and second delay echoes of the $\mathrm{AC}$ for the experimental conditions corresponding to the maximum error in Fig. 10. The power spectral density (PSD) contained in each AC peak is plotted in Fig. 11(d). Inspecting Figs. 11(a) to 11(c), we observe how the experimental $\mathrm{AC}$ is well fitted for the central peak structure and the left side of the first and second delay echoes. However, the fit does not reproduce correctly the right sides of these delay echoes. We found that this disagreement is common for intermediate feedback strengths, at the transition region from strong to weak chaos, for all the pump currents

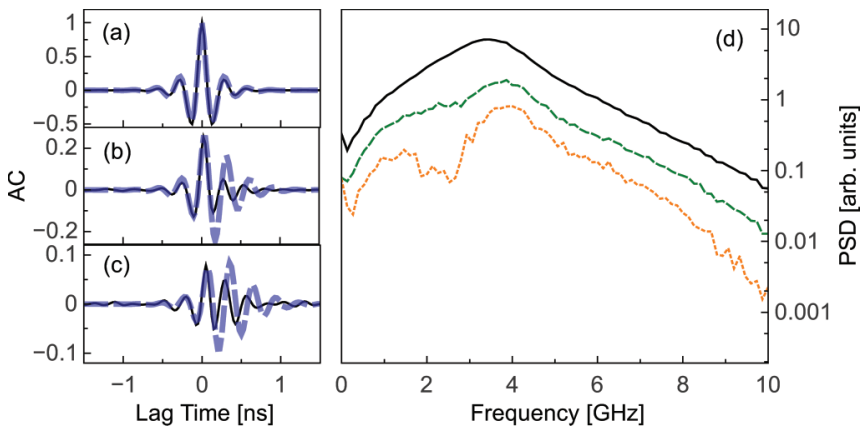

FIG. 11. (Color online) Panels (a) to (c) depict the successive delay echoes. Panel (d) depicts the corresponding power spectral densities. Pump current and rescaled attenuation are $I=16 \mathrm{~mA}$ and $19.1 \mathrm{~dB}$, respectively.

that we have analyzed. Moreover, the significant increase in the fitting error for intermediate feedback strengths suggests that the behavior of the laser deviates the most from linearity around this region. We interpret this to be due to the enhanced nonlinear mixing between the feedback-induced dynamics and the relaxation oscillations frequency occurring at these feedback conditions [17]. As shown in Fig. 10, the fitting error suggests that the linear stochastic model cannot capture the nonlinear response of the laser in the strong chaos regime.

The discrepancy between linear model and experimental AC originates from the fact that multiple dominant frequencies are actually present in our laser systems. This becomes evident from closer inspection of Fig. 11(d), where the power spectral density of the first and second delay echoes exhibit bimodality. This is a signature of the coexistence of two distinctly decaying frequencies that compete in the feedback dynamics. In fact, this feature characterizes the point where all the fitted frequencies in Fig. 6(a) diverge after rescaling. The presence of a second dominant frequency does not seem to have a significant influence on the remaining parameters extracted from the fit.

From Fig. 6(a), it is clear that the divergence between these two frequencies increases with the feedback strength. We can therefore conclude that an extended linear model containing two frequency components with a different damping could overcome this issue for intermediate to strong feedbacks. However, we restrict ourselves here to the simplest linear model, which is accurate for most of the experimental regimes, as this allows for the interpretation of the extracted parameters in the context of a semiconductor laser subject to delayed feedback.

\section{FINAL REMARKS AND CONCLUSIONS}

We have shown that the AC of the intensity of a delayed feedback semiconductor laser can, at least in the long delay limit, be approximated by a linear stochastic model with delay in a wide range of injection currents and feedback strengths. The fit to the linear stochastic model allows for the extraction of certain parameters of the semiconductor laser dynamics, e.g., the relaxation oscillations frequency and damping. Furthermore, the relation of parameters $\phi / \omega$ precisely reproduces the shift in time of the maximum of the $\mathrm{AC}$ at first delay echo. 
The model captures only linear properties of the dynamics in the same way as surrogate data would do. Therefore, the nonlinear mappings and properties like correlation dimension or entropy are not captured by the model. However, we find that the main limitations to fit the experimental $\mathrm{AC}$ with the linear model seem to be related to the necessity to include a second frequency in the model. We identify two possible physical origins for this second frequency depending on the dynamical regime. For low feedback strengths, it can originate from to the presence of anharmonics of the relaxation frequency in the undamped relaxation oscillations regime. For intermediate and strong feedbacks, it can be due to the competition between the relaxation oscillations frequency and the frequency shift introduced by the feedback, which can be of the same order [17].

In this manuscript, we have illustrated how the properties of the AC relate to the dynamical regime of the delayed feedback laser. In particular, we have derived damping $\alpha$ and phase shift $\phi$ from first principles. We argue that this relationship between AC properties and dynamical regime has a broad validity in nonlinear dynamical systems with delay. In this context, it has been shown that the $\mathrm{AC}$ properties of a single delay-coupled system serve as a predictor to the $\mathrm{AC}$ properties of delay-coupled systems $[16,43]$. Therefore, the insights obtained by the linear model can also serve as a tool to interpret the dynamical regimes found in delay-coupled systems.

\section{ACKNOWLEDGMENTS}

The authors acknowledge fruitful discussions with S. Yanchuk. This work was supported by Ministerio de Economía y Competitividad (MINECO, Spain) and Fondo Europeo del Desarrollo Regional (FEDER) via the Project No. TEC2012-36335 (TRIPHOP) and by Comunitat Autònoma de les Illes Balears via Grups Competitius. T.J. acknowledges support by a fellowship within the Postdoc-Programme of the German Academic Exchange Service (DAAD).

\section{APPENDIX A: PARAMETERS FOR THE SIMULATION OF THE LANG-KOBAYASHI EQUATIONS}

The constants, as listed in Table I, were used in the simulation of the LK equations. Figure 12 depicts an exemplary fit of the AC calculated from simulations of the LK model. The

TABLE I. Constants used in the simulation of the LK equations. Values are taken from Ref. [8].

\begin{tabular}{lcl}
\hline \hline Parameter & Symbol & \multicolumn{1}{c}{ value } \\
\hline Linewidth enhancement factor & $\alpha_{H}$ & 2.5 \\
Differential optical gain & $G_{\mathrm{N}}$ & $2.142 \times 10^{4} \mathrm{~s}^{-1}$ \\
Laser frequency & $\omega_{0}$ & $2 \pi c / 1550 \mathrm{~nm}$ \\
Pump current relative to $J_{\text {th }}$ & $p$ & 1.32 \\
Threshold pump current & & \\
of solitary laser & $J_{\text {th }}$ & $\gamma N_{\text {sol }}$ \\
Carrier decay rate & $\gamma$ & $0.909 \times 10^{9} \mathrm{~s}^{-1}$ \\
Carrier number of solitary laser & $N_{\text {sol }}$ & $1.707 \times 10^{8}$ \\
Cavity decay rate & $\Gamma$ & $0.357 \times 10^{12} \mathrm{~s}^{-1}$ \\
Ext. cavity delay time & $\tau$ & $75.25 \mathrm{~ns}$ \\
\hline \hline
\end{tabular}

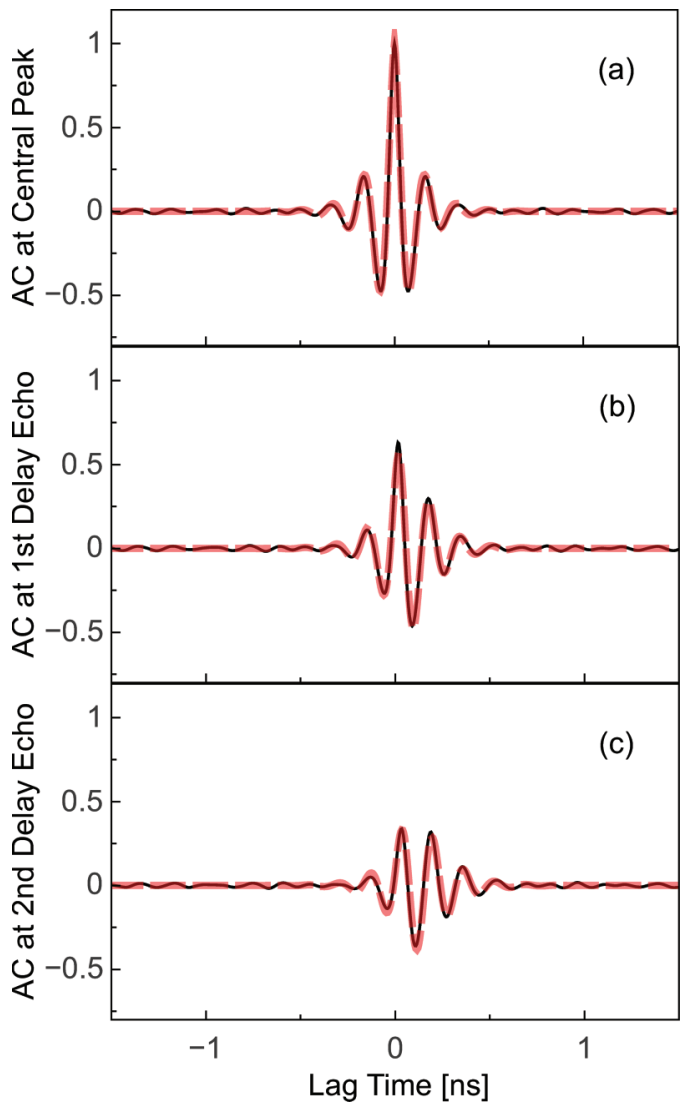

FIG. 12. (Color online) The central peak (a) and the first two delay echoes [(b) and (c), respectively] of the AC calculated from simulations of the LK model at a pump current of $p=1.32$ and a rescaled feedback strength of $46.29 \mathrm{~ns}^{-1}$. The solid lines represent the numeric data and the dashed lines correspond to the fitted analytic expressions for the linear stochastic model.

fitted analytic curves accurately reproduce the numerical AC. Both AC are largely showing the same behavior as obtained from the experimental trajectories.

In the LK equations, the linewidth enhancement factor $\alpha_{H}$ describes the coupling between the real and imaginary parts of the electric field. As such, it reflects one of the main nonlinearities of the semiconductor laser. Although such a parameter is not present in the linear model of the AC, we have checked numerically that the linear model captures the features of the autocorrelation function of the laser in the range $\alpha_{H}=2, \ldots, 6$. However, the fitting error increases with increasing $\alpha_{H}$.

\section{APPENDIX B: CALCULATION OF THE AUTOCORRELATION PHASE AT THE UNDAMPING POINT OF THE RELAXATION OSCILLATIONS}

We rewrite the LK equations (10) in terms of the intensity $I=|\mathcal{E}|^{2}$ and optical phase $\Phi=\arg (\mathcal{E})$,

$$
\begin{aligned}
& \dot{I}=G_{N} n I+2 \kappa \sqrt{I I_{\tau}} \cos \left(\Phi-\Phi_{\tau}\right), \\
& \dot{\Phi}=\frac{\alpha_{H}}{2} G_{N} n+\kappa \sqrt{\frac{I_{\tau}}{I}} \sin \left(\Phi_{\tau}-\Phi\right), \\
& \dot{n}=(p-1) \gamma N_{\mathrm{sol}}-\gamma n-\left(\Gamma+G_{N} n\right) I .
\end{aligned}
$$


The ECMs are given by the steady-state condition $\dot{I}=0, \dot{n}=$ $0, \dot{\Phi}=\omega$, from which follows

$$
\begin{aligned}
I & =\frac{(p-1) \gamma N_{\mathrm{sol}}-\gamma n}{\Gamma+G_{N} n}, \\
n & =-\frac{2 \kappa}{G_{N}} \cos \omega \tau \\
\omega & =-\kappa \sqrt{1+\alpha_{H}^{2}} \sin \left(\omega \tau+\arctan \alpha_{H}\right) .
\end{aligned}
$$

Solutions for which $\cos \left(\omega \tau+\arctan \alpha_{H}\right)<0$ are called antimodes, and they are unstable for any feedback strength. The other solutions are called modes, and they destabilize for increasing feedback through a Hopf bifurcation [31]. The minimal linewidth mode has a frequency closest to the frequency of the solitary laser. In the limit of long delays, it is determined by $\omega \tau=-\arctan \alpha_{H}$ [44]. To calculate its stability, we assume a small perturbation $(\delta I, \delta \Phi, \delta n) \propto e^{\lambda t}$ around the minimal linewidth mode. The stability is then determined by the roots of the characteristic equation,

$$
\operatorname{det}\left[A+e^{-\lambda \tau} B-\lambda \mathbb{1}\right]=0 .
$$

with the partial Jacobians $A=\partial(\dot{I}, \dot{\Phi}, \dot{n}) / \partial(I, \Phi, n)$ and $B=$ $\partial(\dot{I}, \dot{\Phi}, \dot{n}) / \partial\left(I_{\tau}, \Phi_{\tau}, n_{\tau}\right)$

$$
\begin{aligned}
A & =\left(\begin{array}{ccc}
G_{N} n+\kappa \cos \omega \tau & -2 \kappa I \sin \omega \tau & G_{N} I \\
\frac{\kappa}{2 I} \sin \omega \tau & -\kappa \cos \omega \tau & \frac{\alpha}{2} G_{N} \\
-\left(\Gamma+G_{N} n\right) & 0 & -\gamma-G_{N} I
\end{array}\right), \\
B & =\left(\begin{array}{ccc}
\kappa \cos \omega \tau & 2 \kappa I \sin \omega \tau & 0 \\
-\frac{\kappa}{2 I} \sin \omega \tau & \kappa \cos \omega \tau & 0 \\
0 & 0 & 0
\end{array}\right) .
\end{aligned}
$$

The phase shift between $\delta I$ and $\delta I_{\tau}$ is given by $\varphi=\operatorname{Im}(\lambda \tau)$. Using the pseudocontinuous spectrum approach [45] for large delays, we assume $\lambda=i \mu+\gamma / \tau$. The characteristic equation
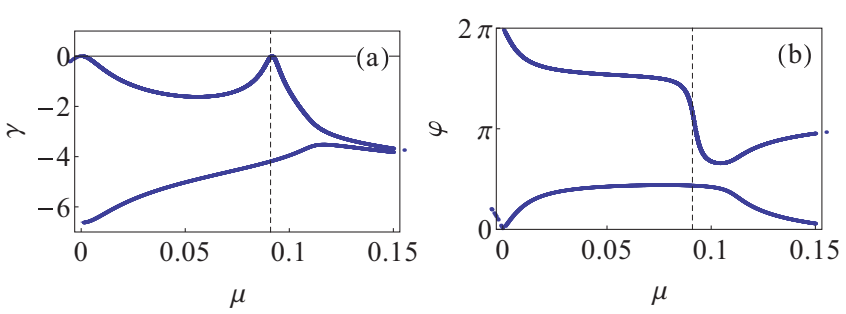

FIG. 13. (Color online) Solutions of the characteristic equation (dots) together with the pseudocontinuous spectrum (lines). Left panel: $\gamma=\Re(\lambda) \tau$ touches the imaginary axis at the bifurcation point, which is indicated with a vertical dashed line. Right panel: $\varphi=\Im(\lambda) \tau$. Parameters are as follows: $\alpha_{H}=2.5, \tau=75.25 \mathrm{~ns}, p=1.32$, and $\kappa_{c}=0.99 \mathrm{~ns}^{-1}$.

can then, in lowest order of $\tau^{-1}$, be rewritten as

$$
\operatorname{det}[A+Y(\mu) B-i \mu \mathbb{1}]=0
$$

with $Y(\mu)=e^{-i \mu \tau-\gamma}$. It is possible to formally solve this equation for $Y(\mu)$. In the complex plane the solutions of the characteristic equation are densely located on the curve $(\mu, \gamma(\mu) / \tau)$, with $\gamma(\mu)=-\ln |Y(\mu)|$. At the bifurcation point $\kappa_{c}$ the pseudocontinuous spectrum touches the imaginary axis at $\mu= \pm \mu_{c} \neq 0$. The phase shift is then given by $\varphi=\arg \left(Y\left(\mu_{c}\right)\right)$, and we identify this phase shift with the phase extracted from the AC function [see Eqs. (3) and (5)]. This is based on the realistic assumption that, slightly above the undamping point of the RO, the dynamics is still mostly governed by the properties of the minimum linewidth mode. Figure 13 shows real and imaginary part of the pseudocontinuous spectrum at the undamping point $\kappa_{c}$. The mode corresponding to $\mu=0$ is the neutral mode (or Goldstone mode) of the system. Around $\mu=0.09$ we find a resonancelike effect, which indicates the most unstable oscillating mode. As soon as $\kappa$ exceeds $\kappa_{c}$, the associated value $\gamma\left(\mu=\mu_{c}\right)$ crosses the horizontal axis. The corresponding phase shift $\varphi$ is located close to $\pi$ and is shown in Fig. 8 .
[1] G. Orosz, R. E. Wilson, R. Szalai, and G. Stepan, Phys. Rev. E 80, 046205 (2009).

[2] M. C. Mackey and L. Glass, Science 197, 287 (1977).

[3] M. B. Elowitz and S. Leibler, Nature (London) 403, 335 (2000).

[4] G. Stepan, Phil. Trans. R. Soc. A 367, 1059 (2009).

[5] A. Argyris, D. Syvridis, L. Larger, V. Annovazzi-Lodi, P. Colet, I. Fischer, J. Garcia-Ojalvo, C. R. Mirasso, L. Pesquera, and K. A. Shore, Nature 438, 343 (2005).

[6] M. C. Soriano, J. García-Ojalvo, C. Mirasso, and I. Fischer, Rev. Mod. Phys. 85, 421 (2013).

[7] I. Fischer, O. Hess, W. Elsäßer, and E. Göbel, Phys. Rev. Lett. 73, 2188 (1994).

[8] V. Ahlers, U. Parlitz, and W. Lauterborn, Phys. Rev. E 58, 7208 (1998).

[9] B. Dorizzi, B. Grammaticos, M. Le Berre, Y. Pomeau, E. Ressayre, and A. Tallet, Phys. Rev. A 35, 328 (1987).

[10] V. S. Anishchenko, G. A. Okrokvertskhov, T. E. Vadivasova, and G. I. Strelkova, New J. Phys. 7, 76 (2005).
[11] G. Giacomelli, R. Meucci, A. Politi, and F. T. Arecchi, Phys. Rev. Lett. 73, 1099 (1994).

[12] C. Masoller, Chaos 7, 455 (1997).

[13] G. Giacomelli, F. Marin, and M. Romanelli, Phys. Rev. A 67, 053809 (2003).

[14] M. C. Soriano, M. Yousefi, J. Danckaert, S. Barland, M. Romanelli, G. Giacomelli, and F. Marin, IEEE J. Select. Top. Quant. Electron. 10, 998 (2004).

[15] M. Sondermann and T. Ackemann, Opt. Express 13, 2707 (2005).

[16] G. Van der Sande, M. C. Soriano, I. Fischer, and C. R. Mirasso, Phys. Rev. E 77, 055202(R) (2008).

[17] X. Porte, M. C. Soriano, and I. Fischer, Phys. Rev. A 89, 023822 (2014).

[18] R. N. Bracewell, The Fourier Transform and Its Applications, (McGraw-Hill, New York, 1978).

[19] S. Heiligenthal, T. Jüngling, O. D’Huys, D. A. Arroyo-Almanza, M. C. Soriano, I. Fischer, I. Kanter, and W. Kinzel, Phys. Rev. E 88, 012902 (2013). 
[20] A. Uchida, K. Amano, M. Inoue, K. Hirano, S. Naito, H. Someya, I. Oowada, T. Kurashige, M. Shiki, S. Yoshimori, K. Yoshimura, and P. Davis, Nat. Photonics 2, 728 (2008).

[21] I. Reidler, Y. Aviad, M. Rosenbluh, and I. Kanter, Phys. Rev. Lett. 103, 024102 (2009).

[22] N. Oliver, M. C. Soriano, D. W. Sukow, and I. Fischer, Opt. Lett. 36, 4632 (2011).

[23] J. Zhang, Y. Wang, M. Liu, L. Xue, P. Li, A. Wang, and M. Zhang, Opt. Express 20, 7496 (2012).

[24] W. Just et al., J. Phys. A: Math. Gen. 34, 3199 (2001).

[25] M. C. Mackey and I. G. Nechaeva, Phys. Rev. E 52, 3366 (1995).

[26] The parameters $\omega, \Delta, \beta$, and $\alpha$ have been rescaled dividing the original extracted parameters by a factor $\sqrt{\frac{I}{I_{t h}}-1}$.

[27] R. W. Tkach and A. R. Chraplyvy, J. Lightwave Technol. 4, 1655 (1986).

[28] D. Lenstra, B. Verbeek, and A. Den Boef, IEEE J. Quant. Electron. 21, 674 (1985).

[29] J. S. Cohen, F. Wittgrefe, M. D. Hoogerland, and J. P. Woerdman, IEEE J. Quant. Electron. 26, 982 (1990).

[30] R. Lang and K. Kobayashi, IEEE J. Quant. Electron. 16, 347 (1980).

[31] S. Yanchuk and M. Wolfrum, SIAM J. Appl. Dyn. Syst. 9, 519 (2010).

[32] M. J. Bünner, A. Kittel, J. Parisi, I. Fischer, and W. Elsässer, Europhys. Lett. 42, 353 (1998).
[33] R. Hegger, M. J. Bünner, H. Kantz, and A. Giaquinta, Phys. Rev. Lett. 81, 558 (1998).

[34] V. S. Udaltsov, J. P. Goedgebuer, L. Larger, J. B. Cuenot, P. Levy, and W. T. Rhodes, Phys. Lett. A 308, 54 (2003).

[35] M. D. Prokhorov, V. I. Ponomarenko, A. S. Karavaev, and B. P. Bezruchko, Physica D 203, 209 (2005).

[36] M. Siefert, Phys. Rev. E 76, 026215 (2007).

[37] D. Rontani, A. Locquet, M. Sciamanna, and D. S. Citrin, Opt. Lett. 32, 2960 (2007).

[38] D. Rontani, A. Locquet, M. Sciamanna, D. S. Citrin, and S. Ortin, IEEE J. Quant. Electron. 45, 879 (2009).

[39] L. Zunino, M. C. Soriano, I. Fischer, O. A. Rosso, and C. R. Mirasso, Phys. Rev. E 82, 046212 (2010).

[40] J. G. Wu, G. Q. Xia, X. Tang, X. D. Lin, T. Deng, L. Fan, and Z. M. Wu, Opt. Express 18, 6661 (2010).

[41] R. Nguimdo, M. Soriano, and P. Colet, Opt. Lett. 36, 4332 (2011).

[42] S. Heiligenthal, T. Dahms, S. Yanchuk, T. Jüngling, V. Flunkert, I. Kanter, E. Schöll, and W. Kinzel, Phys. Rev. Lett. 107, 234102 (2011).

[43] M. C. Soriano, V. Flunkert, and I. Fischer, Chaos 23, 043133 (2013).

[44] B. Tromborg and J. Mørk, IEEE J. Quant. Electron. 26, 642 (1990).

[45] S. Yanchuk and P. Perlikowski, Phys. Rev. E 79, 046221 (2009). 